\title{
The CMS muon barrel drift tubes system commissioning
}

\author{
G. Abbiendi ${ }^{\text {h }}$, N. Amapane ${ }^{\mathrm{a}, \mathrm{b}}$, C. Battilana ${ }^{\mathrm{h}, \mathrm{i}}$, C. Fernandez Bedoya ${ }^{\mathrm{d}}$, R. Bellan ${ }^{\mathrm{a}, \mathrm{b}}$, M. Bellato ${ }^{\mathrm{e}}$,
}

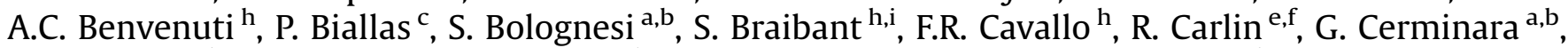
M. Chamizo ${ }^{\text {d }}$, E. Conti ${ }^{\text {e }}$, G.M. Dallavalle ${ }^{\text {h }}$, D. Dattola ${ }^{a}$, G. Dellacasa ${ }^{a}$, M.C. Fouz ${ }^{\text {d, }}$, Frangenheim ${ }^{c}$, P. Giacomelli ${ }^{\text {h }}$, M. Giunta ${ }^{\text {h }}$, F. Gonella ${ }^{\mathrm{e}}$, A. Gresele ${ }^{\mathrm{e}, \mathrm{g}}$, L. Guiducci $^{\text {h,i }}$, K. Hoepfner ${ }^{\mathrm{c}}$, E. Jacobi ${ }^{\mathrm{c}}$, S. Marcellini ${ }^{\text {h }}$, C. Mariotti ${ }^{\text {a }}$, S. Maselli a , G. Masetti ${ }^{\text {h,i }}$, A.T. Meneguzzo ${ }^{\text {e,f }}$, G. Mila ${ }^{\text {a,b }}$, V. Monaco ${ }^{\text {a,b }}$, A. Montanari ${ }^{\text {h }}$, F. Montecassiano ${ }^{\mathrm{e}}$, F.L. Navarria ${ }^{\mathrm{h}, \mathrm{i}}, \mathrm{M}$, Nervo ${ }^{\mathrm{a}, \mathrm{b}}, \mathrm{F}$. Odorici ${ }^{\mathrm{h}}$, M. Passaseo ${ }^{\mathrm{e}}$, A. Parenti $^{\mathrm{e}, \mathrm{f}}$, A. Perrotta $^{\text {h }}$, J. Puerta ${ }^{\text {d }}$, H. Reithler ${ }^{c}$, P. Ronchese ${ }^{\text {e,f }}$, T. Rovelli ${ }^{\text {h,i }}$, R. Sacchi ${ }^{\text {a,b }}$, G.P. Siroli h,i , M. Sowa ${ }^{c}$,

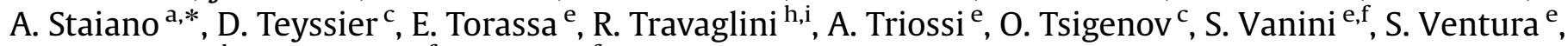
C. Villanueva ${ }^{\mathrm{d}}$, M. Zanetti ${ }^{\mathrm{e}, \mathrm{f}}$, P. Zotto ${ }^{\mathrm{e}, \mathrm{f}}$

a Istituto Nazionale di Fisica Nucleare, via P.Giuria 1, 10125 Torino, Italy

' Universita' di Torino, Torino, Italy

${ }^{\mathrm{c}}$ RWTH Aachen University, III. Physikalisches Institut A, Aachen, Germany

d CIEMAT, Madrid, Spain

e Istituto Nazionale di Fisica Nuclear sez. di Padova, Italy

${ }^{\mathrm{f}}$ Universita' di Padova, Padova, Italy

' Universita' di Trento, Povo, Italy

${ }^{\mathrm{h}}$ Istituto Nazionale di Fisicac Nucleare sez. di Bologna, Italy

' Universita' di Bologna, Bologna, Italy

\section{A R T I C L E I N F O}

\section{Available online 26 August 2008}

Keywords:

CMS

Muon

Drift tubes

Barrel system

\section{A B S T R A C T}

The CMS muon barrel drift tubes system has been recently fully installed and commissioned in the experiment. The performance and the current status of the detector are briefly presented and discussed. (c) 2008 Elsevier B.V. All rights reserved.

\section{Introduction}

The CMS detector [1] is close to completion, and starting from summer 2008 it will be ready to be operated on LHC. A very important section of the apparatus is the muon system [2], which is composed of a barrel part (the drift tubes system) and a forward part (the cathode strip chambers system). Both tracking sections, the barrel and the endcap, have also first-level triggering capabilities, which are also duplicated with the resistive plate chamber barrel and endcap systems. In this paper, after a short description of the barrel drift tubes system, we will review the status of the commissioning of this section of the muon system.

\section{The CMS barrel drift tube system}

Each of the five wheels of the CMS barrel muon detector [1] is made of four stations (labelled MB1, MB2, MB3 and MB4) forming

\footnotetext{
* Corresponding author. Tel.: +39011670 7332; fax: +390116699579.

E-mail address: amedeo.staiano@to.infn.it (A. Staiano).
}

concentric cylinders around the beam line, and arranged in 12 sectors: the three inner ones consist of $60 \mathrm{drift}$ chambers each, the outer one of 70 (see Fig. 1). The total number of sensitive wires is about 172000 . The wire length, around $2.4 \mathrm{~m}$ in the chambers measuring in the $r$, $\phi$ projection, is constrained by the longitudinal segmentation of the iron barrel yoke. The half-transverse dimension of the drift cell, i.e. the maximum path and time of drift, is $21 \mathrm{~mm}$ (corresponding to a drift time of $380 \mathrm{~ns}$ in $85 \% \mathrm{Ar} 15 \% \mathrm{CO}_{2}$ ).

A drift tube chamber is made of three (or two in the outer layers) superlayers, each made by four layers of rectangular drift cells staggered by half a cell. The wires in the two outer SLs are parallel to the beam line and provide the track measurement in the magnetic bending plane $(r, \phi)$. In the inner SL, the wires are orthogonal to the beam line and measure the $z$ position along the beam. The third $z$-measuring SL is not present in the fourth layer of chambers, which therefore measures only the $\phi$ coordinate. A muon coming from the interaction point encounters a $\Phi$ SL first, passes through the honeycomb plate, then crosses the $Z$ SL and the second $\Phi$ SL. Redundancy of information (needed at high momenta $(>40 \mathrm{GeV})$ due to the possible presence of electromagnetic cascades accompanying the parent muon) is obtained by 
having four layers of separated drift cells per station. The tube cross-section dimensions are $13 \mathrm{~mm} \times 42 \mathrm{~mm}$.

A single SL gives excellent time tagging capability, with a time resolution of a few nanoseconds. This capability provides local, stand-alone and efficient bunch-crossing identification and therefore first-level triggering capability. The time tagging is delayed by a constant amount of time equal to the maximum possible drift time, which is determined by the size of the tube, the electrical field and the gas mixture. The time resolution is largely independent of the track angle.

The bunch-crossing tagging is performed independently in each of the three SLs, by fast pattern-recognition circuitry directly mounted on dedicated supports (called minicrates [3]) on each chamber. This circuit delivers, together with the bunch-crossing assignment, the position of the center of gravity of the track segment and its angle in the SL reference system, with the precision of $1.5 \mathrm{~mm}$ and $20 \mathrm{mrad}$, respectively. This information is used by the first-level muon trigger for the time and transverse momentum assignment. In parallel to this, RPC first-level trigger detectors are directly attached to each DT chamber providing a fully independent and complementary trigger system [1].

The mechanical precision of the construction of a chamber is dictated by the aim to achieve the global resolution in $(r-\phi)$ of $100 \mu \mathrm{m}$. This figure makes the MB1 chamber (the innermost layer) precision comparable to the multiple scattering contribution up to $p_{\mathrm{T}}=200 \mathrm{GeV}$. The $100-\mu \mathrm{m}$ target chamber resolution is achieved with eight track points measured in the two $(r-\Phi) \mathrm{SL}$.

The cell design makes use of five electrodes: one anode wire, two field-shaping strips and two cathode strips, the two latter electrodes being built with the same technique, a $50-\mu \mathrm{m}$-thick aluminum tape deposited on top of a $100-\mu \mathrm{m}$-thick insulating mylar tape glued on the aluminum structural parts of the cells.

\section{Chamber assembly and installation}

The 250 chambers, which form the complete CMS Barrel DT System, were assembled in four production laboratories (RWTH Aachen, CIEMAT Madrid, INFN Padova and INFN Torino), which

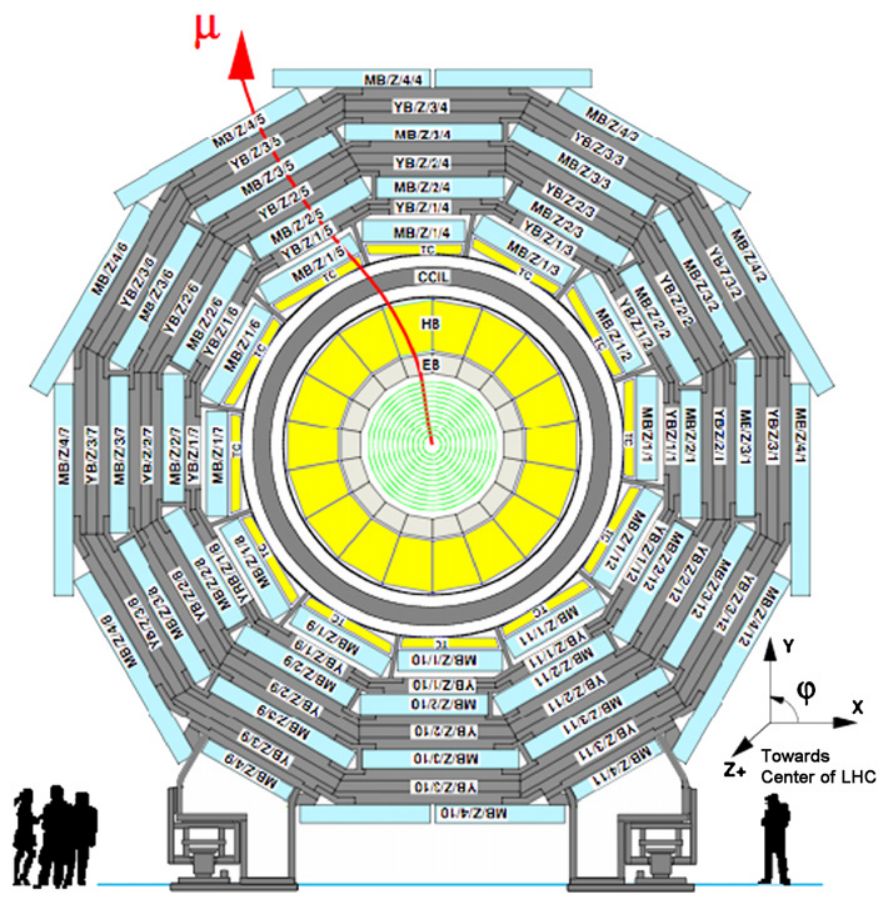

Fig. 1. Transverse view of a CMS barrel yoke wheel. shared the work following the four different typologies of chambers (from the inner layer MB1 produced in Aachen to, following the same order, the outer layer MB4 produced in Torino). In parallel to chamber assembly, parts assembly and electronics design and test were carried out in other laboratories (INFN Bologna-IHEP Protvino, INFN Torino-JINR Dubna, RWTH Aachen, IHEP Beijing, CIEMAT Madrid and INFN Padova). Construction started in January 2002 and was completed in June 2006. All chambers were fully tested in the production laboratories with large cosmic data samples, allowing full debugging of each chamber before expedition to CERN. Once at CERN, all chambers were dressed with their final electronics (minicrates and final gas system), retested on a cosmic test stand, surveyed on a dedicated alignment bench in order to determine wire positions with respect to the external reference marks of the general CMS barrel alignment system (built by the groups of Universidad de Cantabria, Santander and KFKI Budapest), and coupled to the RPC detectors. Installation in the five yoke wheels of the CMS detector started on surface in July 2004 and was completed in cavern in October 2007.

\section{Detector commissioning}

The commissioning of the DT Barrel System has been a longlasting process running at various stages in parallel to chamber production and installation. Besides the dedicated test beam runs taken on prototypes and on final detectors prior and during chamber construction [4-8], chamber commissioning was performed through the following phases:

1. test detectors with cosmics at production sites, with final FE electronics, temporary trigger and readout electronics, test gas tightness, efficiency, dead, noisy channels and resolution;

2. full dressing with final onboard trigger and readout electronics (minicrates). Test again as in point 1 prior installation, couple to RPC and survey;

3. installation and full test of each chamber with cosmics, with temporary cabling and local data acquisition system;

4. Magnet Test and Cosmic Challenge (MTCC) with 5\% of fully operational detectors installed and the superconducting solenoid magnet ramped at nominal $4 \mathrm{~T}$ field during summer 2006;

5. full commissioning of equipped wheels with all chambers and final CMS DAQ, HV, LV, detector control system and cabling in the cavern;

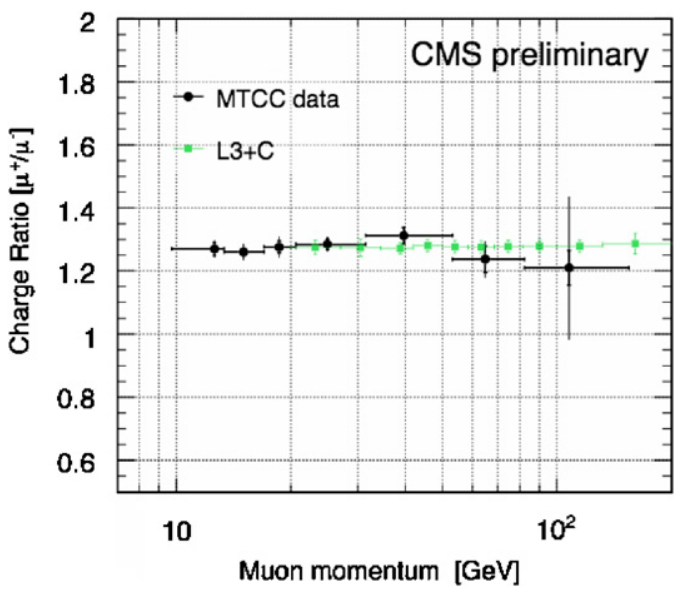

Fig. 2. Positive to negative cosmic muon ratio as a function of $p_{\mu}$ 
6. Global Run, with full integration of the DT system in CMS trigger and data acquisition chain.

The test described in phase 4 , although being performed only on $5 \%$ of the apparatus (bottom sectors 10,11 of wheels $\mathrm{YB}+2$ and

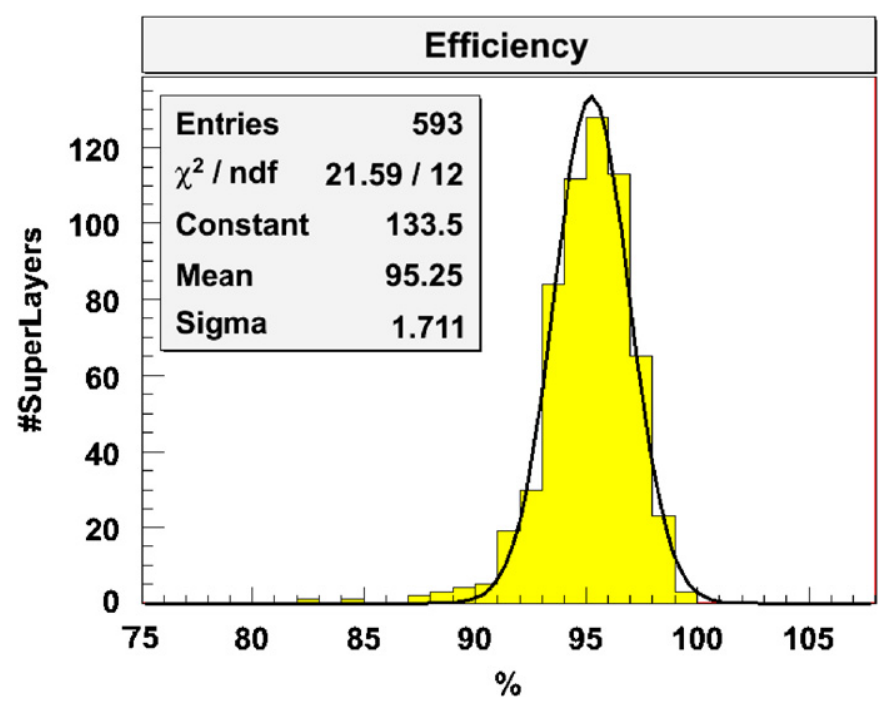

Fig. 3. Summary plot of overall system efficiency. Inefficiencies include also cell geometry effects (as dead regions covered by the cathode I-beams).
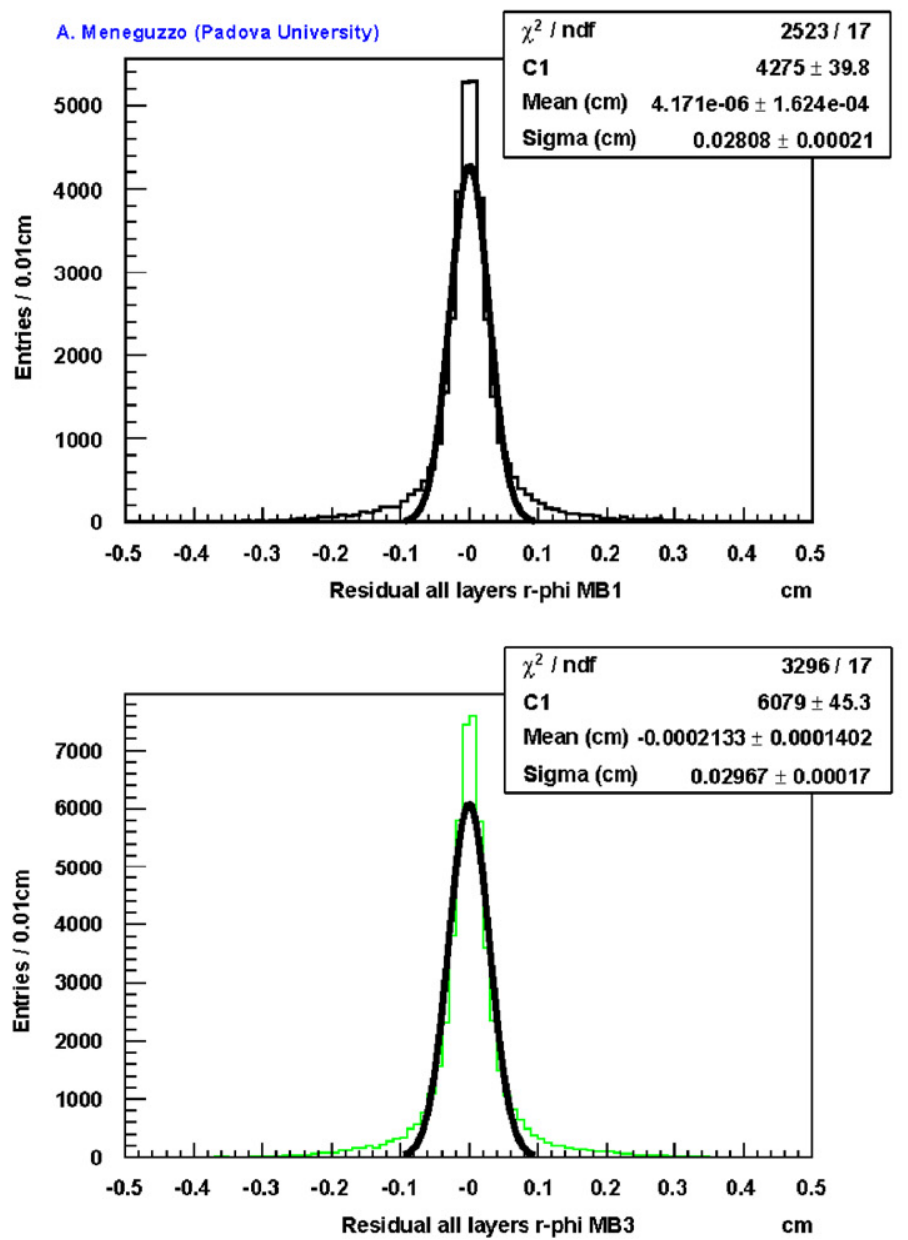

sector 10 in wheel $\mathrm{YB}+1$ ), led to the achievement of several important milestones:

- Chamber performance: check HV behaviour, drift velocity, position resolution and efficiency, check the effect of fringe field on chamber performance and compare to chamber behaviour with B-field off. In particular it was confirmed that the smearing of the trigger BX identification in the outer $\theta$ region of MB1 chambers in the +2 wheel (where the drift cell distortion is maximal) amounts to a maximum of $0.3 \mathrm{BX}$, which is well within trigger specifications. The complete readout chain (with final readout system and Front-End Driver (FED)) was successfully integrated. Tests of HV and LV were done with the final setup.

- Trigger: test of the setup with final hardware up to the DT Track Finder level was done providing cosmic-ray trigger to CMS.

- Software: interplay with databases (condition, configuration $\mathrm{DB})$ to retrieve configuration settings/store MC conditions was tested with full integration of all software components in CMSSW, the official CMS software framework (data unpacking, data quality monitoring, reconstruction, visualization).

As an interesting outcome of the large amount of cosmic data collected with the magnet on, a measurement of the ratio of $R=N\left(\mu^{+}\right) / N\left(\mu^{-}\right)$was obtained [9] and compared with existing data, as shown in Fig. 2.

The test of all installed chambers, as described in phase 5 , has been fully completed, allowing full characterization of the system.
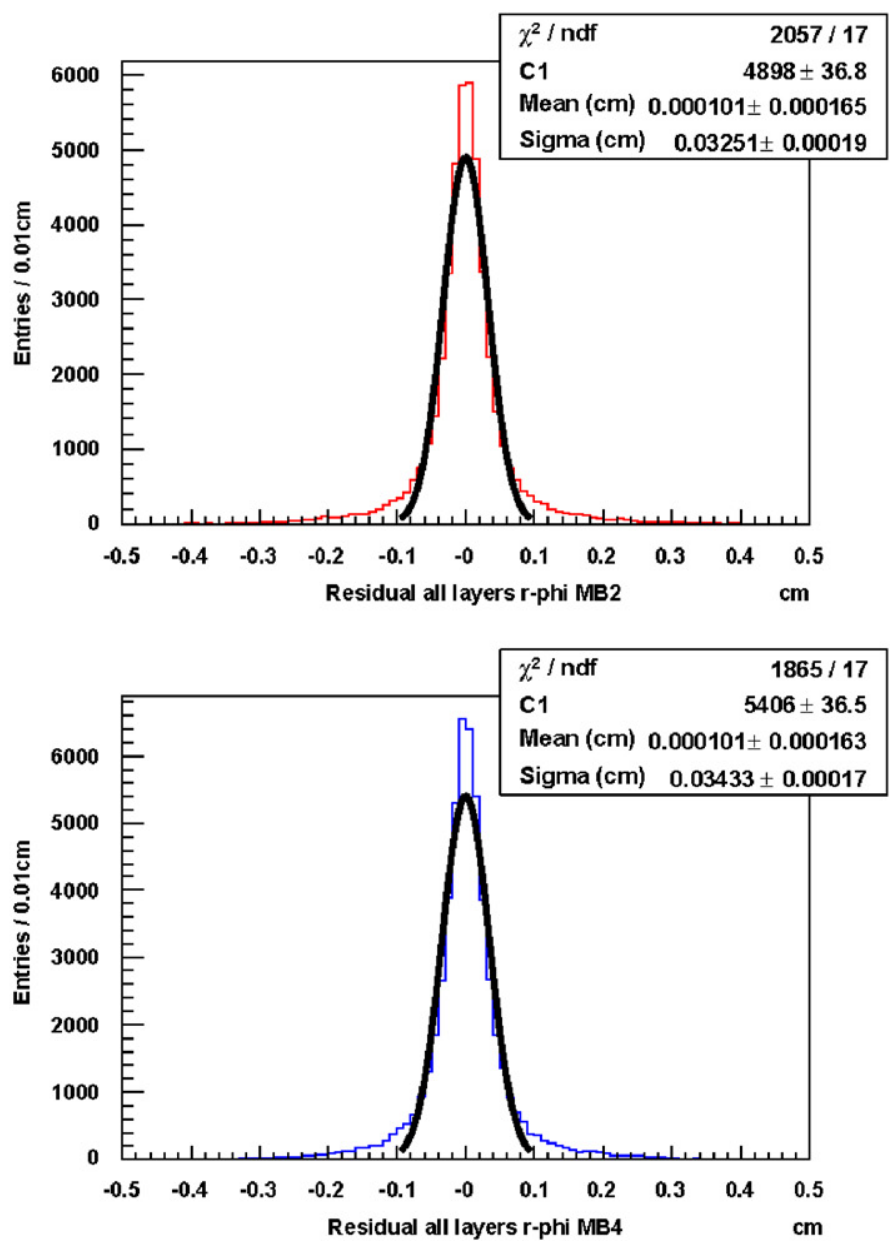

Fig. 4. Mean resolution for the four chamber layers (MB1-MB4). The best SL resolution was obtained on dedicated test beams [4] with $\sigma=170 \mu \mathrm{m}$. 


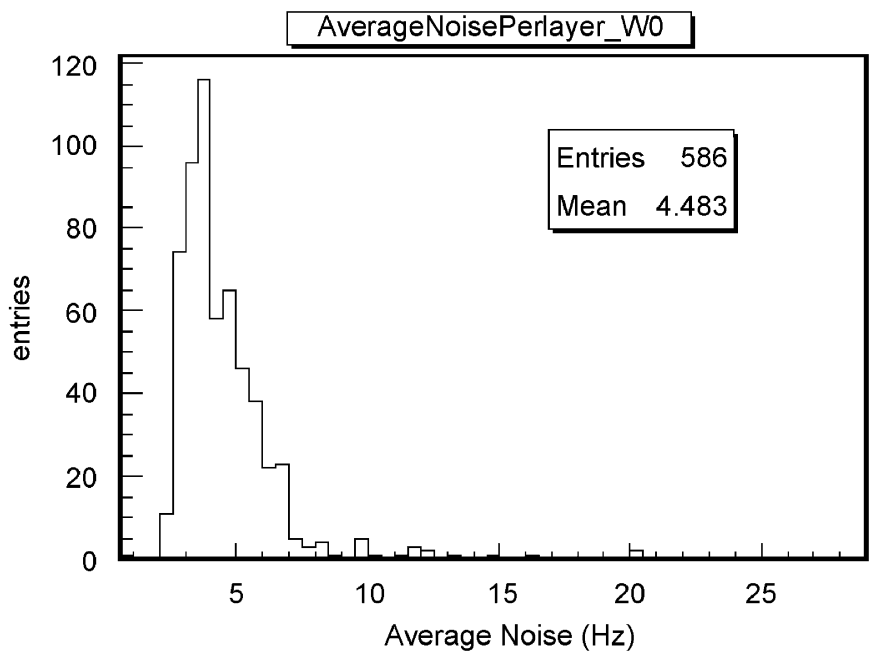

Fig. 5. Average noise/layer for wheel 0 , after subtraction of channels with noise rate $>200 \mathrm{~Hz}$.

This was obtained after fine-tuning of the trigger system timing, which was optimized for cosmic tagging. The underground cosmic rates detected by single-chamber triggering ranges from 3 to $30 \mathrm{~Hz}$ depending on the sector orientation. Good cosmic statistics can be therefore collected to measure cell efficiencies of all 172,000 channels. Cell efficiency is flat both with respect to channel numbers and for the different typologies and dimensions of chambers. Its average value is shown in Fig. 3.

The overall amount of dead channels is 311, which corresponds to $0.2 \%$ of the whole system. Single-cell resolution can be measured, as shown in Fig. 4 for the four types of chambers, averaging over all SL layers.

Dedicated runs during phase 5 of the commissioning with random triggers were collected in order to evaluate the noise level in all channels. The average noise/layer in wheel YBO is shown in Fig. 5.

This figure is very similar for the five wheels, which have approximately the same amount of noisy channels and with an average noise/layer level ranging from 4.2 to $4.7 \mathrm{~Hz}$.

The final phase of the commissioning, phase 6 , is common to all sub-detectors. It is a test of full integration of all sub-detectors with the central CMS DAQ and triggering system, with the full exercise of online and offline databases handling and Monte Carlo production. In the last CMS Global Run in November 2007, $100 \%$ of barrel hadron calorimeter, 50\% of forward hadron calorimeter, $20 \%$ of the DT system (the central wheel 0 ) and 10\% of RPC and Electromagnetic Calorimeter (ECAL) were integrated in the run (see Fig. 6).

The exercise, with an integrated statistics of $\sim 10 \mathrm{M}$ events, accomplished several goals, namely the synchronization of ECAL

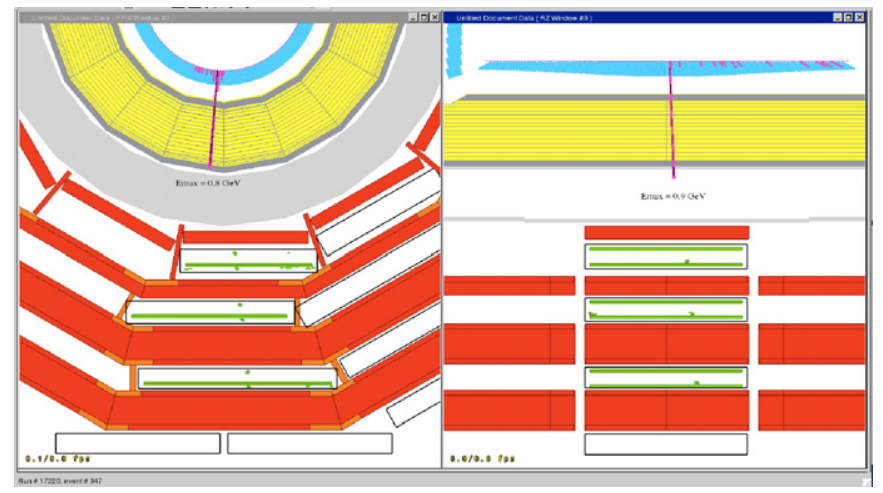

Fig. 6. Event triggered by the DT system during November 2007 Global Run with a cosmic muon tagged on sector 10 and a release of $\sim 1-\mathrm{GeV}$ energy on ECAL.

and DT systems with the CMS clock, the relative DT trigger and DT readout synchronization, the integration of the DT trigger with the CMS global trigger, the integration of the ECAL and DT readout with the global CMS DAQ system, with the commissioning of the FED systems of all participating sub-detectors.

\section{Conclusions}

The commissioning of the five wheels of the full DT system has been completed. This corresponds to the full test and integration of 250 chambers, divided into 60 sectors, which are currently integrated in the CMS trigger, DAQ and offline framework. CMS Global Runs have proven the DT trigger and readout systems to be well integrated, with good reliability and stability.

Currently work is ongoing on inter-wheel synchronization and fine synchronization with the RPC and CSC systems. The system is ready for the CMS full Global Run at $0 \mathrm{~T}$ with the participation of all sub-detectors, which will be run in May 2008, while in July 2008 the CMS Global Run will be run with the CMS solenoid ramped at its nominal field of $4 \mathrm{~T}$.

\section{References}

[1] S. Chatrchyan et al., The CMS Collaboration, The CMS Experiment at CERN LHC, JINST 3 (2008) S08004.

[2] CMS Collaboration, The Muon Project, Technical Design Report, CERN/LHCC 97-32, 1997.

[3] C. Fernandez Bedoya, et al., IEEE Trans. Nucl. Sci. NS-52 (2005) 944.

[4] C. Albajar, et al., Nucl. Instr. and Meth. A 525 (2004) 465.

[5] M. Aguilar-Benitez, et al., Nucl. Instr. and Meth. A 416 (1998) 243.

[6] M. Aguilar-Benitez, et al., Nucl. Instr. and Meth. A 480 (2002) 658.

[7] P. Arce, et al., Nucl. Instr. and Meth. A 534 (2004) 441.

[8] M. Aldaya, et al., Nucl. Instr. and Meth. A 579 (2007) 951.

[9] M. Aldaya, et al., Measurement of the charge ratio of cosmic muons using CMS data, CMS note submitted PNI 592/200. 